\title{
How Do I Rely on Thee, Smartphone?
}

\section{Let me count the ways. Seriously, the smartphone has become the Swiss Army knife of computing and communication. You can make phone calls. What is more}

important, you can use it to exercise any of the millions of applications supported by the Internet. We order products and services. We upload photos we take with the smartphone's increasingly high-resolution camera. They have onboard software for photo and video processing. We use them to start our cars and, in some cases, summon them (Tesla, for instance). We text, tweet, and email our friends. We make and watch YouTube and TikTok videos. We received access codes for two-factor authentication of our accounts. We make and receive payments. We keep track of our exercises and monitor our glucose levels (with suitable add-on gear). We connect to myriad devices with BlueTooth so our smartphones become controllers or sources of music. Indeed, in connection with other devices, our smartphones are becoming part of our remote medical care and monitoring systems.

Our smartphones translate written and even spoken languages. For deaf users, it transcribes speech in 100 languages and generates speech from text. We use them to do character recognition and language translation (think menus). We use them to navigate because they know where they are with precision, thanks to global positioning systems. We use them to surf the Internet and World Wide Web. I am sure I have missed your favorite applications, but you can fill in the blanks. However, and there is always a "however," there are some downsides to this cornucopia of utility wrapped up in a pocket-sized device.

What could possibly go wrong? Setting aside obvious annoyances like inadvertent phone calls (some of my friends call this "butt dialing"), which may end up being recorded on the receiving end's voicemail, the deep dependence we have on the reliable functioning of this infinitely versatile device worries me. I have experienced cascade failures when my smartphone battery is dead, or I cannot get a 3G-4G-5G signal. There are times when I must put my phone in a box to isolate it during confidential meetings and can't therefore use it as a second factor for strong authentication. The more we rely on the reliable functioning of our smartphones, the more things can go wrong when they

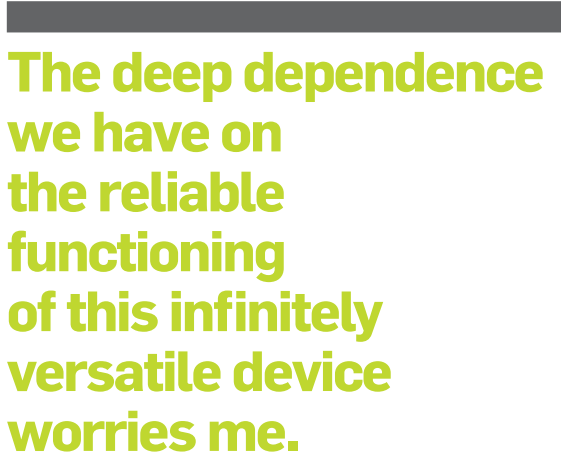

don't work as advertised. There are many ways in which these devices can fail. Remember the poem about "For want of a nail, the kingdom was lost?"

Here's a small example: I could not log into my account because the smartphone was out of range, and I could not get the second factor. Since I could not log in, I could not read my email that had an urgent message with further reference to a file I needed access to in order to conclude a business transaction. You get the idea. So, what's to be done? We need alternate backup procedures to invoke when our primary methods that rely on the smartphone don't work. This is, of course, a smart engineering concept in any case, but given the massive and increasing dependency we place on smartphones, I conclude that we should be thinking about alternatives such as Web-based applications reachable from a laptop, for example. Or printed backup authenticators for two-factor logins. I am sure you can think of other examples. The important point is not to assume $100 \%$ reliability of access to and use of a smartphone, as appealing as that might seem.

Vinton G. Cerf is vice president and Chief Internet Evangelist at Google. He served as ACM president from 2012-2014. 